\title{
Hyperuricemia in Hypertension and Chronic Kidney Disease: Risk Factors, Prevalence and Clinical Correlates: A Descriptive Comparative Study
}

\author{
Peter K. Uduagbamen 1*, John 0. Ogunkoya², Abdallah 0. AdebolaYusuf ${ }^{3}$, A. T. Oyelese ${ }^{4}$, \\ Chukwuyerem I. Nwogbe ${ }^{1}$, Chiamaka J. Ofoh ${ }^{1}$, Chukwuma Anyaele ${ }^{1}$
}

\author{
${ }^{1}$ Division of Nephrology and Hypertension, Department of Internal Medicine, Ben Carson (Snr) School of Medicine, Babcock \\ University/Babcock University Teaching Hospital, Ilishan-Remo, Nigeria \\ ${ }^{2}$ Pulmonology Unit, Department of Internal Medicine, Ben Carson (Snr) School of Medicine, Babcock University/Babcock \\ University Teaching Hospital, Ilishan-Remo, Nigeria \\ ${ }^{3}$ Division of Radiodiagnosis, Department of Surgery, Ben Carson (Snr) School of Medicine, Babcock University/Babcock \\ University Teaching Hospital, Ilishan-Remo, Nigeria \\ ${ }^{4}$ Department of Hematology and Blood Transfusion, Ben Carson (Snr) School of Medicine, Babcock University/Babcock \\ University Teaching Hospital, Ilishan-Remo, Nigeria \\ Email: * petr.uduagbamen@gmail.com
}

How to cite this paper: Uduagbamen, P.K., Ogunkoya, J.O., AdebolaYusuf, A.O., Oyelese, A.T., Nwogbe, C.I., Ofoh, C.J. and Anyaele, C. (2021) Hyperuricemia in Hypertension and Chronic Kidney Disease: Risk Factors, Prevalence and Clinical Correlates: A Descriptive Comparative Study. International Journal of Clinical Medicine, 12, 386-401.

https://doi.org/10.4236/ijcm.2021.129035

Received: August 23, 2021

Accepted: September 25, 2021

Published: September 28, 2021

Copyright $\odot 2021$ by author(s) and Scientific Research Publishing Inc. This work is licensed under the Creative Commons Attribution International License (CC BY 4.0).

http://creativecommons.org/licenses/by/4.0/

(c) (i) Open Access

\begin{abstract}
Introduction: Uric acid is a product of purine metabolism and elevated serum concentration are very common in, and linked with hypertension and chronic kidney disease, conditions associated with heavy health burden and cardiovascular complications particularly in sub Sahara Africa. An assessment of factors relating hyperuricemia to hypertension and chronic kidney disease would therefore be necessary as way of mitigating the poor quality of life, morbidity and mortality associated with these diseases in low income nations. Methods: A single centre, descriptive comparative study in which the demographic, clinical and laboratory data of hypertensive and non-dialyzed chronic kidney disease (CKD) patients were analyzed. Serum biochemical parameters with uric acid, hematocrit and urine dip strip protein were assessed. Predictors of hyperuricemia were determined using multivariate analysis. Results: One hundred and thirty nine hypertensives and 69 CKD were studied. The mean age of the participants was $54.3 \pm 11.7$ years, hypertensives $(52.9 \pm 15.7$ years $)$ and CKD $(57.3 \pm 16.1$ years $)$. Both groups had more males, $\mathrm{P}=0.8$. Majority $(78.3 \%)$ of the $\mathrm{CKD}$ cohorts had stage 4 or 5 (non-dialyzed) disease. The systolic and diastolic blood pressure, creatinine and uric acid were lower in hypertension than in CKD, $\mathrm{P}=0.07, \mathrm{P}=0.05, \mathrm{P}<$ 0.001 and $\mathrm{P}=0.004$ respectively. The hematocrit, albumin and GFR were
\end{abstract}


higher in HTN than CKD, $\mathrm{P}<0.001, \mathrm{P}<0.001$ and $\mathrm{P}<0.001$ respectively. The prevalence of hyperuricemia was $56.2 \%$. The mean uric acid was $505.9 \pm$ $23.6 \mathrm{mmol} / \mathrm{L}, 3827 \pm 10.5 \mathrm{mmol} / \mathrm{L}$ for hypertensive and $755.9 \pm 14.8 \mathrm{mmol} / \mathrm{L}$ for CKD, $\mathrm{P}<0.001$. The prevalence of systolic HTN, proteinuria, hypoalbuminemia and anemia were 51\%,75\%,46\% and 59\%, and were higher in males. Hyperuricemia was related to advancing age, proteinuria, elevated creatinine, hypoalbuminemia, anemia and hypertriglyceridemia. Proteinuria (OR-4.66, 95\% CI-2.42 - 9.65), elevated creatinine (OR-3.12, 95\% CI-2.40 - 6.92), hypoalbuminemia (OR-2.92, 95\% CI-1.83 - 5.78) and anemia (OR-4.01, 95\% CI-3.78 - 7.99) independently predicted hyperuricemia. Conclusion: Hyperuricemia is commoner in CKD than hypertension and was higher in males and positively correlated with the blood pressure, proteinuria and creatinine, but negatively related to hematocrit, albumin and glomerular filtration rate. Independent predictors of hyperuricemia were proteinuria, elevated creatinine, hypoalbuminemia and anemia. Measures are needed to prevent and treat hyperuricemia to reduce the health burden associated with hypertension and CKD.

\section{Keywords}

Hyperuricemia, Hypertension, Chronic Kidney Disease, Anemia, Hypoalbuminemia, Inflammation, Atherosclerosis, Reactive Oxygen Specie

\section{Introduction}

Hyperuricemia has been identified as a risk factor for hypertension (HTN), and the occurrence and progression of chronic kidney disease (CKD) including cardiovascular events [1]. Hypertension and CKD have been on the increase worldwide with worsening socioeconomic burden, just as the prevalence of hyperuricemia in hypertension and CKD is reported to be on the increase leading to a faster progression of hypertension to $\mathrm{CKD}$, and CKD progression to end stage kidney disease (ESRD) [2]. The prevalence CKD in sub-Sahara Africa (SSA) is about $13.9 \%$ and the prevalence of hyperuricemia in CKD in SSA is reported to be $15.2 \%-67 \%$ [3] [4] [5].

Uric acid (UA) as a product of purine nucleotides catabolism, is known to be more commonly elevated in HTN and CKD than in health [2]. Common sources of UA include animal proteins and fructose containing diet and drinks [3]. Although a definitive causative relationship has not been established between hyperuricemia and HTN or CKD, the associations between hyperuricemia and HTN, and with CKD are reported to be mediated through chronic inflammatory changes with renal microvascular injury involving the endothelium induced by the activation of the renin angiotensin aldosterone system (RAAS) [6]. This occurs mostly in the intracellular and intravascular spaces and an end point of this inflammatory state is endothelial injury, release of vasoactive cytokines, atherosclerosis and increased cardiovascular risk profile [7]. Uric acid as a weak acid is 
also reported to have strong anti-oxidant properties in the extracellular (EC) space [8].

Hyperuricemia is reported to mitigate the inflammatory injury associated with many chronic inflammatory and degenerative diseases like Alzheimers' disease, Parkinson's disease and chronic obstructive lung diseases (COPD) [9] [10] [11]. Nieto et al. [12] reported that in atherosclerotic patients, hyperuricemia induces a compensatory reduction in vascular oxidative damage with increased proximal tubular sodium absorption, as found in hyperinsulinemia. The world health organization (WHO) reported that over one billion people have hyperuricemia worldwide accounting for $13 \%$ of death and is implementing a preventive program aimed at reducing the global prevalence of hyperuricemia by $25 \%$ by the year 2025 [13]. Hyperuricemia is reported to be commoner in urban than rural communities and this has been attributed to the lifestyle pattern in urban settings associated with dietary indiscretion, particularly high intake of animal protein and fructose (sweetened) containing drinks [5].

Apart from uric acid stones, hyperuricemia is known to induce gout in addition to renal inflammation [9]. The occurrence of hyperuricemia from declining renal losses is often time, due to the inability of the compensatory increases in gastrointestinal losses to keep serum levels within normal [14]. Hyperuricemia has a synergistic effect on kidney function decline which could lead to a worsening metabolic acidosis (MA) and declining hematocrit, although the bimodal profile of serum albumin concentration (regarding increases as an inflammatory marker and reduction from increased losses, both prominent features of CKD) makes it difficult to draw outright conclusions, based only on its blood levels [15]. Considering the association between hyperuricemia and conditions like hypertension, $\mathrm{CKD}$ and cardiovascular disease, some authors have assessed the impact of uric acid lowering agents on renal and cardiovascular function [16] [17]. However, a generalized causal relationship is still being debated, it might still be a sound clinical verdict to assume the renal, cardiac and vascular toxicity of hyperuricemia, thereby, cautiously preventing it and its associated health burden.

Hyperuricemia is well reported locally and internationally, however, literature is scares concerning a comparative assessment of its associations with hypertension, and $\mathrm{CKD}$, regarding determinants and clinical correlates. We hypothesize that hyperuricemia is common among hypertensives but more so in CKD. We compared hyperuricemiain hypertension and CKD.

\section{Materials and Methods}

This was a singer center hospital based descriptive, comparative study carried out at the Nephrology and Hypertension Clinic of Babcock University Teaching Hospital, Ilishan-Remo, Nigeria, from August 2019 to January 2021. Two hundred and eight (139 hypertensives and 69 non-dialyzed CKD) participants, sixteen years or older, attending the nephrology and hypertension clinic were consecutively recruited after obtaining informed consent. Chronic kidney disease 
was defined according to the KDOQI 2012 criteria [18]. Participants were not taking uric acid lowering agents at the time of sample collection. All participants had a kidney ultrasound scan and participants with kidney length less than $9 \mathrm{~cm}$ were classified as having CKD [19].

\section{Exclusion Criteria}

Patients with kidney graft, pelvic tumors, infections and HWCKD with proteinuria were excluded. Infections were ruled out by: the absence of fever $(\mathrm{T}<$ $37.4^{\circ} \mathrm{C}$ ) or leucocytes or nitrites on urine analysis and, with a normal ranged white cell count (WCC) and differentials from full blood count test. Hypertensives without $\mathrm{CKD}$ with any of the following conditions: diabetes, sickle cell anemia, liver disease, heart failure, proteinuria on urinalysis or kidney length $<9$ $\mathrm{cm}$ on kidney ultrasound, other conditions that impacted negatively on kidney function, were excluded.

The sample size was calculated using the prevalence of hyperuricemia in a similar study [20].

Data was taken from history and patients' case notes and variables retrieved were age, gender, family history of hypertension and CKD, type and etiology of CKD.

Participants' height was taken without shoes, caps or head gear and weight on very light clothing using standardized scales and the body mass index (BMI) was calculated. The blood pressure (BP) was taken with a mercury sphygmomanometer (ACCOSON, England) with an appropriate standard cuff, after at least, 5 minutes rest.

Five milliliters of venous blood was collected from a peripheral vein into a $\mathrm{Li}$ thium heparin bottle for estimation of serum sodium, potassium, bicarbonate, chloride, urea, creatinine and uric acid. Serum biochemical analysis was determined using an autoanalyzer (Roche Diagnostics GmbH, Mannheim Germany). The creatinine based glomerular filtration rate (GFR) was calculated using the Chronic Kidney Disease Epidemiology Collaboration (CKD-EPI) formula. About 1 milliliter of blood was also taken for determination of the hematocrit using a hematocrit centrifuge. An on-the-spot urinalysis was carried out on all participants' urine samples. Participants' fasting blood glucose (FBS) was determined using an Omron glucometer with glucose oxidase impregnated stripe.

\section{Definitions}

Hypeuricemia: Males $>0.42 \mathrm{mmol} / \mathrm{L}$, Females $>0.36 \mathrm{mmol} / \mathrm{L}[20]$.

Hypertension: $\geq 140 / 90 \mathrm{mmHg}$ [21].

Diabetes: Medical records confirming disease or history of use of antidiabetic drugs.

Dyslipidaemia: Total cholesterol $\geq 6.21 \mathrm{mmol} / \mathrm{L}$.

Low-density lipoprotein cholesterol (LDL) $>4.14 \mathrm{mmol} / \mathrm{L}$

High-density lipoprotein cholesterol (HDL) $<1.03 \mathrm{mmol} / \mathrm{L}$

Triglycerides $\geq 1.69 \mathrm{mmol} / \mathrm{L}$ [22] 
Anemia: Hematocrit < 33\% [23]

Proteinuria: dip strip protein $\geq 1+[24]$

Hypoalbuminemia: serum albumin $<35 \mathrm{mg} / \mathrm{dL}$ [25]

eGFR (CKD-EPI)-ml/min/1.73 $\mathrm{m}^{2}$ [26]

Data analysis was carried out SSPS 22. Continuous variables are presented as mean with standard deviation and compared using student's t-test while categorical variables are presented as proportions and frequencies and compared using Chi-square or fisher's exact test. The P-value $<0.05$ was considered statistically significant. After univariate analyses, variables with $\mathrm{p}<0.025$ were included as adjustment variables in multivariate analyses to determine variables that predicted hyperuricemia. Missing data where excluded from the analysis pairwise. This study was approved by the Babcock University Human Research Ethics Committee (NHREC/24/01/2018 and BUHREC501/19).

\section{Results}

Two hundred and eight participated (139 hypertensives and 69 CKD). The mean age of all participants was $54.3 \pm 11.7$ years, with hypertensives without CKD $(52.9 \pm 15.7$ years $)$ and CKD (57.3 \pm 16.1 years $)$ respectively. Males made up $64.9 \%, 64.0 \%$ and $66.7 \%$ of all participants, hypertensives and CKD respectively, $\mathrm{P}=0.8$. Three $(4.3 \%)$ of the CKD cohort had stage 2 disease, $5(7.2 \%)$ in stage $3 a, 7(10.1 \%)$ in stage 3b, $13(18.8 \%)$ in stage 4 and $41(59.4 \%)$ in stage 5 (non-dialytic). As participants advanced in age, the prevalence of hypertension and CKD increased, $\mathrm{P}=0.13$. The mean $\mathrm{BMI}$ was lower in HTN than in CKD, $\mathrm{P}$ $=0.08$. The systolic and diastolic blood pressure, serum potassium, urea, creatinine and uric acid were lower in hypertension than in $C K D, P=0.07, P=0.05, P$ $<0.001, \mathrm{P}<0.001, \mathrm{P}<0.001$ and $\mathrm{P}=0.004$ respectively (Table 1 ). The serum sodium, bicarbonate, chloride, hematocrit, albumin and GFR were higher in HTN than in CKD, $\mathrm{P}<0.001, \mathrm{P}=0.028, \mathrm{P}=0.005, \mathrm{P}<0.001, \mathrm{P}<0.001$ and $\mathrm{P}<$ 0.001 respectively. The mean serum uric acid for study population was $505.9 \pm$ $23.6 \mathrm{mmol} / \mathrm{L}$. There was no statistical difference between the mean blood glucose of participants with hypertension and, with $\mathrm{CKD}, \mathrm{P}=0.7$.

The mean uric acid was $505.9 \pm 23.6 \mathrm{mmol} / \mathrm{L}, 3827 \pm 10.5 \mathrm{mmol} / \mathrm{L}$ in HWCKD and $755.9 \pm 14.8 \mathrm{mmol} / \mathrm{L}$ for $\mathrm{CKD}, \mathrm{P}<0.001$. The serum uric acid was higher in males and was positively correlated with the age, BMI, the systolic BP, level of proteinuria and serum creatinine, $\mathrm{P}<0.001, \mathrm{P}<0.001, \mathrm{P}=0.001, \mathrm{P}<$ 0.001 and $\mathrm{P}<0.001$ respectively, but was negatively correlated with serum bicarbonate, albumin, and hematocrit, $\mathrm{P}<0.001, \mathrm{P}<0.001$ and $\mathrm{P}<0.001$ respectively (Table 2). Derangements of serum biochemical and hematological parameters were associated with greater differences in the uric acid concentration between HWCKD and CKD.

The prevalence of hyperuricemia, systolic HTN, proteinuria, hypoalbuminemia and anemia in all participants were 56.3\%, 51.0\%, 75.0\%, 46.2\% and 59.1\% respectively (Table 3 ), and were all higher in males than females.

Univariate analysis (Table 4) showed that advancing age, male gender, prote- 
inuria, elevated creatinine, hypoalbuminemia, anemia and hypertriglyceridemia were associated with hyperuricemia. Multivariate analysis (Table 5) however showed only proteinuria (OR-4.66, 95\% CI-2.42 - 9.65, P $<0.001)$, elevated creatinine $(\mathrm{OR}-3.12,95 \% \mathrm{CI}-2.40-6.92, \mathrm{P}=0.002)$, hypoalbuminemia $(\mathrm{OR}-2.92,95 \% \mathrm{CI}-1.83-5.78, \mathrm{P}=0.01)$ and anemia $(\mathrm{OR}-4.01,95 \% \mathrm{CI}-3.78$ - 7.99, $\mathrm{P}=0.001)$ independently predicted hyperuricemia.

Table 1. Sociodemographic, clinical and laboratory characteristics of participants.

\begin{tabular}{|c|c|c|c|c|}
\hline \multirow{3}{*}{ Variables } & Total & Hypertension & CKD & \multirow{3}{*}{ P-value } \\
\hline & $\mathrm{N}=208(\%)$ & $\mathrm{N}=139(\%)$ & $\mathrm{N}=69(\%)$ & \\
\hline & Mean \pm SD & Mean \pm SD & Mean \pm SD & \\
\hline \multicolumn{5}{|l|}{ Sex } \\
\hline Males & 135 (64.9) & $89(64.0)$ & $46(66.7)$ & 0.8 \\
\hline Females & $73(35.1)$ & $50(36.0)$ & $23(33.3)$ & \\
\hline \multicolumn{5}{|l|}{ Age, yrs } \\
\hline Mean & $54.3 \pm 11.6$ & $52.9 \pm 15.7$ & $57.3 \pm 16.1$ & 0.04 \\
\hline$<65$ & $99(47.6)$ & $68(48.9)$ & $31(44.9)$ & 0.05 \\
\hline$>65$ & $109(52.4)$ & $71(51.1)$ & $38(55.1)$ & \\
\hline \multicolumn{5}{|l|}{$\mathrm{BMI}, \mathrm{kg} / \mathrm{m}^{2}$} \\
\hline$<25.0$ & $88(47.1)$ & $69(49.6)$ & $29(42.0)$ & 0.08 \\
\hline$>25.0$ & $110(52.9)$ & $70(50.4)$ & $40(58.0)$ & \\
\hline \multicolumn{5}{|l|}{ Systolic BP, mmHg } \\
\hline$<140$ & $102(51.0)$ & $70(50.4)$ & $32(46.4)$ & 0.07 \\
\hline$>140$ & $106(49.0)$ & $69(49.6)$ & $37(53.6)$ & \\
\hline \multicolumn{5}{|l|}{ Diastolic BP, mmHg } \\
\hline$<90$ & $96(46.2)$ & $65(46.8)$ & $31(44.9)$ & 0.05 \\
\hline$>90$ & $112(53.8)$ & $74(53.2)$ & $38(55.1)$ & \\
\hline Proteinuria, $(>15 \mathrm{mg} / \mathrm{dL})$ & $156(75.0)$ & $91(65.5)$ & $65(94.2)$ & $<0.001$ \\
\hline Sodium, mmol/L & $134.5 \pm 5.5$ & $136.9 \pm 5.2$ & $132.8 \pm 8.4$ & $<0.001$ \\
\hline Potassium, mmol/L & $4.1 \pm 1.0$ & $3.9 \pm 0.6$ & $4.4 \pm 1.02$ & $<0.001$ \\
\hline Chloride, $\mathrm{mmol} / \mathrm{L}$ & $99.8 \pm 3.7$ & $101.0 \pm 8.7$ & $97.5 \pm 8.0$ & 0.005 \\
\hline Bicarbonate, mmol/L & $25.8 \pm 12.6$ & $27.2 \pm 16.3$ & $23.0 \pm 14.1$ & 0.028 \\
\hline Calcium, mmol/L & $2.3 \pm 1.0$ & $2.5 \pm 1.2$ & $2.0 \pm 0.4$ & 0.03 \\
\hline Phosphate, $\mathrm{mmol} / \mathrm{L}$ & $1.8 \pm 0.6$ & $1.6 \pm 1.1$ & $2.3 \pm 1.4$ & 0.002 \\
\hline Urea, $\mathrm{mmol} / \mathrm{L}$ & $14.8 \pm 7.3$ & $8.7 \pm 3.0$ & $26.9 \pm 16.4$ & $<0.001$ \\
\hline Creatinine, umol/L & $187.6 \pm 43.1$ & $143 \pm .50 .6$ & $275 \pm .57 .0$ & $<0.001$ \\
\hline $\mathrm{eGFR}, \mathrm{ml} / \mathrm{min}$ & $46.9 \pm 4.9$ & $69.6 \pm 3.4$ & $21.7 \pm 5.8$ & $<0.001$ \\
\hline Uric acid, mmol/L & $505.9 \pm 23.6$ & $382.7 \pm 10.5$ & $755.9 \pm 14.8$ & $<0.001$ \\
\hline Hematocrit, \% & $34.1 \pm 6.6$ & $39.4 \pm 6.8$ & $25.9 \pm 7.9$ & $<0.001$ \\
\hline $\mathrm{RBG}, \mathrm{mmol} / \mathrm{L}$ & $118.8 \pm 11.7$ & $118.4 \pm 14.3$ & $111.9 \pm 11.8$ & 0.7 \\
\hline Albumin, mg/dL & $39.3 \pm 8.3$ & $42.1 \pm 8.6$ & $33.7 \pm 6.9$ & $<0.001$ \\
\hline
\end{tabular}

CKD—chronic kidney disease, BMI-body mass index, BP-blood pressure, eGFR—estimated glomerular filtration ratio, $\mathrm{RBG}$-random blood glucose. 
Table 2. Comparison between the uric acid in hypertension and chronic kidney disease.

\begin{tabular}{|c|c|c|c|c|}
\hline & Total & Hypertension & CKD & \\
\hline & Uric acid & Uric acid & Uric acid & \\
\hline \multirow[t]{3}{*}{ Variables } & $\mathrm{mmol} / \mathrm{L}$ & $\mathrm{mmol} / \mathrm{L}$ & $\mathrm{mmol} / \mathrm{L}$ & $\mathrm{P}$-value \\
\hline & $\mathrm{N}=208(\%)$ & $\mathrm{N}=139(\%)$ & $\mathrm{N}=69(\%)$ & \\
\hline & Mean \pm SD & Mean \pm SD & Mean \pm SD & \\
\hline Mean & $505.9 \pm 23.6$ & $3827 \pm 10.5$ & $755.9 \pm 14.8$ & $<0.001$ \\
\hline \multicolumn{5}{|l|}{ Sex } \\
\hline Males & $516.8 \pm 22.6$ & $422.5 \pm 21.5$ & $825.9 \pm 33.7$ & $<0.001$ \\
\hline Females & $452.0 \pm 84.8$ & $329.4 \pm 78.4$ & $648.0 \pm 91.8$ & 0.001 \\
\hline \multicolumn{5}{|l|}{ Age, years } \\
\hline$<65.0$ & $444.7 \pm 5$ & $308.0 \pm 21.4$ & $513.3 \pm 23.6$ & 0.05 \\
\hline$>65.0$ & $555.7 \pm 33.2$ & $466.2 \pm 28.5$ & $853.5 \pm 33.7$ & $<0.001$ \\
\hline \multicolumn{5}{|l|}{$\mathrm{BMI}, \mathrm{kg} / \mathrm{m}^{2}$} \\
\hline$<25.0$ & $496.3 \pm 77.5$ & $355.4 \pm 36.8$ & $612.5 \pm 67.4$ & 0.04 \\
\hline$>25.0$ & $513.1 \pm 34.3$ & $402.0 \pm 67.7$ & $824.5 \pm 86.2$ & $<0.001$ \\
\hline \multicolumn{5}{|c|}{ Systolic BP, mmHg } \\
\hline$<140$ & $473.4 \pm 23.9$ & $356.7 \pm 18.6$ & $727.2 \pm 34.0$ & 0.03 \\
\hline$>140$ & $528.8 \pm 51.5$ & $405.3 \pm 23.6$ & $793.0 \pm 57.2$ & 0.001 \\
\hline \multicolumn{5}{|l|}{ Proteinuria } \\
\hline$>15 \mathrm{mg} / \mathrm{dL}$ & $557.3 \pm 13.7$ & $433.8 \pm 23.6$ & $782.6 \pm 36.3$ & $<0.001$ \\
\hline$<15 \mathrm{mg} / \mathrm{dL}$ & $348.9 \pm 9.9$ & $283.8 \pm 11.5$ & $316.5 \pm 10.6$ & 0.05 \\
\hline \multicolumn{5}{|c|}{ Creatinine, umol/L } \\
\hline$<110$ & $398.6 \pm 18.2$ & $323.7 \pm 9.6$ & $739.7 \pm 24.2$ & $<0.001$ \\
\hline$>110$ & $585.4 \pm 22.4$ & $415 \pm 31.4$ & $769.5 \pm 13.7$ & $<0.001$ \\
\hline \multicolumn{5}{|c|}{ Bicarbonate, $\mathrm{mmol} / \mathrm{L}$} \\
\hline$<22$ & $558.3 \pm 9.5$ & $4162 \pm 22.5$ & $802.1 \pm 11.6$ & $<0.001$ \\
\hline$>22$ & $476.3 \pm 8.8$ & $369.4 \pm 7.9$ & $714.6 \pm 10.9$ & 0.04 \\
\hline \multicolumn{5}{|c|}{ Albumin, mg/dL } \\
\hline$<35$ & $572.1 \pm 10.4$ & $446.3 \pm 11.5$ & $795.8 \pm 14.5$ & $<0.001$ \\
\hline$>35$ & $458.7 \pm 9$ & $351.8 \pm 9.3$ & $728.9 \pm 9.9$ & $<0.001$ \\
\hline \multicolumn{5}{|c|}{ Hematocrit, \% } \\
\hline$<33$ & $532.2 \pm 7.8$ & $426.0 \pm 8.3$ & $867.2 \pm 9.5$ & $<0.001$ \\
\hline$>33$ & $411.9 \pm 7.5$ & $331.7 \pm 11.6$ & $601.4 \pm 12.4$ & 0.001 \\
\hline
\end{tabular}

$\mathrm{CKD} —$ chronic kidney disease, $\mathrm{BMI}=$ body mass index, $\mathrm{BP} \_$blood pressure. 
Table 3. Prevalence of hyperuricemia and its markers in the study population.

\begin{tabular}{ccccc}
\hline Variables & Total & Males & Females & \multirow{2}{*}{ P-value } \\
\cline { 2 - 4 } & $\mathrm{N}=208(\%)$ & $\mathrm{N}=135(\%)$ & $\mathrm{N}=73(\%)$ & \\
\hline Hyperuricemia & $117(56.3)$ & $86(63.7)$ & $31(42.5)$ & 0.08 \\
Systolic hypertension & $106(51.0)$ & $77(57.0)$ & $29(39.7)$ & 0.06 \\
Proteinuria & $156(75.0)$ & $114(84.4)$ & $42(57.5)$ & 0.04 \\
Elevated creatinine & $147(70.7)$ & $110(81.5)$ & $37(50.7)$ & 0.04 \\
Hypoalbuminemia & $96(46.2)$ & $69(51.1)$ & $27(37.0)$ & 0.16 \\
Anemia & $123(59.1)$ & $81(60.0)$ & $42(57.3)$ & 0.62 \\
Low HDL & $107(51.4)$ & $72(53.3)$ & $35(47.9)$ & 0.42 \\
Elevated LDL & $100(48.1)$ & $72(53.3)$ & $28(38.4)$ & 0.06 \\
Hypertriglyceridemia & $111(53.4)$ & $77(57.0)$ & $33(45.2)$ & 0.33 \\
\hline
\end{tabular}

LDL-low density lipoprotein, HDL—high density lipoprotein.

Table 4. Univariate analysis of factors associated with hyperuricemia.

\begin{tabular}{|c|c|c|c|}
\hline \multirow{2}{*}{ Variables } & No Hyperuricemia & Hyperuricemia & \multirow{2}{*}{$\mathrm{P}$-value } \\
\hline & $\mathrm{N}=91$ & $\mathrm{~N}=117$ & \\
\hline Age, years $($ Mean $\pm S D)$ & $33.9 \pm 4.7$ & $63.3 \pm 8.5$ & $<0.001$ \\
\hline Sex & 91 & 117 & 0.03 \\
\hline Males (n, \%) & $49(36.3)$ & $86(63.7)$ & \\
\hline Females (n, \%) & $42(57.5)$ & $31(42.5)$ & \\
\hline Hypertension (n, \%) & $74(53.2)$ & $65(46.8)$ & 0.001 \\
\hline CKD (n, \%) & $17(24.6)$ & $52(75.4)$ & \\
\hline Overweight/Obesity (n, \%) & $34(37.4)$ & $76(65.0)$ & 0.04 \\
\hline Proteinuria (n, \%) & $52(57.1)$ & $104(88.9)$ & $<0.001$ \\
\hline Elevated creatinine, mean & $162.6 \pm 13.6$ & $226.8 \pm 21.3$ & $<0.001$ \\
\hline (range) & $(92.7-184.6)$ & $(118.7-357.9)$ & \\
\hline$(\mathrm{n}, \%)$ & $36(39.6)$ & $111(94.9)$ & \\
\hline Hypoalbuminemia (n, \%) & $17(18.7)$ & $79(67.5)$ & $<0.001$ \\
\hline Anemia (n, \%) & $31(34.1)$ & $92(78.6)$ & $<0.001$ \\
\hline Low HDL (n, \%) & $34(37.4)$ & $73(62.4)$ & 0.07 \\
\hline Elevated LDL (n, \%) & $30(33.0)$ & $70(59.8)$ & 0.05 \\
\hline Hypertriglyceridemia & $32(35.2)$ & $79(67.5)$ & 0.02 \\
\hline
\end{tabular}

CKD—chronic kidney disease, HDL—high density lipoprotein, LDL—low density lipoprotein. 
Table 5. Multivariate analysis of independent predictors of hyperuricemia.

\begin{tabular}{cccc}
\hline Variables & OR & $95 \% \mathrm{CI}$ & P-value \\
\hline Age & 1.17 & $1.04-4.26$ & 0.05 \\
Proteinuria & 4.66 & $2.42-9.65$ & $<0.001$ \\
Elevated creatinine & 3.12 & $2.40-6.92$ & 0.002 \\
Hypoalbuminemia & 2.92 & $1.83-5.78$ & 0.01 \\
Anemia & 4.01 & $3.78-7.99$ & 0.001 \\
Hypertriglycedemia & 1.08 & $0.96-2.01$ & 0.05 \\
\hline
\end{tabular}

OR—odd ratio, CI—confidence interval.

\section{Discussion}

The prevalence of hyperuricemia for all participants in our study was $56.3 \%$, and was higher in CKD than in HWCKD (75.4\% vs $46.8 \%$ ), as it was higher in males than females (63.7\% vs $42.5 \%)$. The prevalence of hyperuricemia in HWCKD mirrors that found by Cannon et al. [27] but higher than the $41.4 \%$ (35\% in males, $43 \%$ in females) found in a nationwide survey of Taiwanese hypertensives [28], higher than the $38.7 \%$ found in the Chinese hypertensive population [29], and much higher than the $14 \%$ reported by Fan et al. [30]. It is however lower than the $59.3 \%$ and $62 \%$ reported by Emokpae et al. [31] among males and female respectively, in a Nigerian hypertensive population.

Apart from variations in the diagnostic cut-off across population segments, differences in the methodology could be contributory. Our prevalence of hyperuricemia in CKD is similar to that that reported in a United State pediatric hypertensive population with $70 \%$. It is higher than the $67 \%$ reported by Doualla et al. [5] in Cameroon, another low income nation, higher than the $47.5 \%$ found by Adejumon et al. [4] in Nigeria, higher than the $60 \%$ found in Italy, and much higher than the $15.2 \%$ reported in Chad. Our study population was made up of recently diagnosed hypertensives, and CKD patients who were not receiving uric acid lowering therapy (ULT). Our facility being a tertiary health care centre commonly receives cases in advance stages of disease. Our inclusion of non-dialytic stage $5 \mathrm{CKD}$ patients also contributed to the high prevalence.

We found a higher prevalence of hyperuricemia in males than females and this is in agreement with findings by Alikor and Makususidi and their respective groups in Nigeria and Wang et al. in China [32] [33] [34]. Hyperuricemia is however reported to be more prevalent in females [28] [31] [35]. The higher prevalence in males in our study could be multifactorial. First, higher estrogen concentrations in females particularly premenopausal, confers on them higher uricosuric ability [32]. Though the use of alcohol and smoking was not assessed in this study, their use (and therefore higher risk for hyperuricemia) are reported to be commoner amongst males than females [32] [36]. The culture-enhancing socioeconomic advantage of men over women in our clime, makes them (men) more likely to take more meat (animal protein) than females [37]. The toxic ef- 
fect of testosterone on the renal tubules further contributes to the greater decline in tubular uric acid secretion associated with increased sodium reabsorption in the proximal tubules [38].

The positive relationship between the age and the uric acid concentration (UAC) mirrors findings by Cameroon, and that by Avram et al. [5] [38] but is not in agreement with findings by Grayson et al. [35] and Lin et al. who reported higher UAC in the younger age groups. Even in health, the physiologic decline in kidney function from middle age is expected to lead to a relative kidney function decline with advancing age resulting in lower uric acid excretion. The relatively smaller volume of total body water (TBW) in the elderly would also contribute to a greater delivery of sodium to the distal tubules and an adaptive increase in sodium absorption coupled with decreased uric acid secretion in the PT [28].

We found a positive correlation between the BMI and UAC, and is in agreement with previous studies. Obesity induces hyperinsulinism associated with increased leptin production. Hyperinsulinemia cause increased absorption of sodium and urate in the proximal tubule leading to hypertension and hyperuricemia [39]. The synergistic effect of declining urate secretion from aging and increase absorption from hyperinsulinemia in our study could explain the higher uric acid in the elderly and in the overweight/obese, a combination that doesn't agree with finding from many previous studies [28] [29] [30].

The positive correlation between the uric acid levels and the blood pressure in our study agrees with previous studies that reported the link between hyperinsulinemia and hypertension. In animal models, hyperuricemia acutely induces increased renin production from the juxtaglomerular apparatus (JGA), suppresses both macula densa nitric oxide synthase (NOS) release and phosphorylation of endothelial nitric oxide (eNOS). The resultant release of reactive oxygen specie (ROS) and vasoconstrictive mediators, with increased sodium reabsorption at the PT lead to hypertension [40]. Continued chronic inflammatory damages from hyperuricemia lead to microvascular injury involving the afferent arterioles, increased smooth muscle reuptake of uric acid, activation of more chronic inflammatory mediators like monocyte chemo attractant protein-1 (MCP-1) and expression of cyclo-oxygenase-2 (COX-2) pathway [41]. These inflammatory processes create a chronic vasoconstrictive vascular bed with ischemia, hypertension, vascular stiffness and eventually, atherosclerosis as reported in the Generation 3 Framinghan study [42]. A cohort study of 3584 Japanese with prehypertension showed that hyperuricemia increased the risk of hypertension [43], Uric acid $>0.410 \mathrm{mmol} / \mathrm{L}$ predicted refractory hypertension in women older than 65 years (OR-3.11, 95\% CI-1.06 - 9.0), independent of CKD [44] Imazu et al. in a systematic review, reported hyperuricemia in $25 \%-40 \%$ of untreated hypertensives and in $70 \%$ of patients with malignant hypertension [45]. We found a positive relationship between proteinuria and the urate levels and this agrees with earlier studies [5] [46]. Proteinuria results, among other conditions, 
from chronic immunologic injury to the glomerular filtration apparatus involving shedding of the endothelial surfaces into sub-epithelial spaces and replacement of the foot processes by continuous bands along the basement membrane. Although we didn't assess albumin loss in this study, it is worth noting that the resultant alteration in barrier selectivity and eventual cupping (fusion) of the cytoplasmic strands of the podocytes (effacement) leads to albuminuria/proteinuria [47]. The higher proteinuria in CKD compared to hypertension would therefore suggest a more intense immunologic response.

We found a positive correlation between hyperuricemia and anemia, as previously reported [48]. Anemia and hyperuricemia could be adjudged to be due to declining kidney function. Our study didn't seek to establish a link between these two, but McAdams DeMarco et al. [48] had reported that hyperuricemia cause anemia from oxidative stress, a feature common in the two conditions, and in CKD. Although the role of the hepatocytes in protein synthesis was not accessed in this study, the positive relationship between hyperuricemia and blood pressure on one hand, and its negative relationship with serum albumin on the other hand tend to favor proteinuria as the major cause of the hypoalbuminemia than reduced hepatic synthesis, in these cohorts. Due to the higher urea and creatinine among the CKD cohort compared with the hypertensives, coupled with the positive correlation between hyperuricemia and serum creatinine, we infer that proteinuria is a more likely cause of hypoalbuminemia than reduced hepatic synthesis in this cohort [49].

The higher degree of proteinuria and dyslipidemia in males in this study agrees with authors that reported that hypoproteinemia activates hepatic lipid synthesis leading to the release of, at times, excessive lipoprotein moieties in the blood (in a bit to maintain normal serum protein) including the more artherogenic forms of LDL [50]. Considering the role of the LDL subunits in atherosclerosis and its relationship with the blood pressure, the higher blood pressure found among the CKD cohorts in this study explain an initiating role for proteinuria in atherosclerosis and hypertension.

It is known that hyperuricemia results from over production and/or decline in excretion of urate. Overall, the positive relationship between hyperuricemia and, aging, elevated creatinine, elevated blood pressure and anemia (correlates of declining kidney function) is more is more suggestive of hyperuricemia arising from reduced excretion than from increased production [51].

This study was not without limitations. We couldn't assess proteinuria through the more sensitive spot Albustix Test. The relatively small sample size would limit the wider applicability of findings. Participants were not followed up to determine a cause and effect relationship between hyperuricemia and target organs or measures. As participants were recently diagnosed and not receiving ULT, the relationship between hyperuricemia and medications was not determined. The strength is in its assemblage of a wide range of variables that could be related, independently or in association to hyperuricemia in hypertension and CKD. 


\section{Conclusion}

Hyperuricemia is very common in hypertension, more so, in CKD, commoner in males, advancing age and higher BMI. Correlates of hyperuricemia such as higher blood pressure, proteinuria and hypoalbuminemia, anemia and reduced kidney function were more prevalent in $\mathrm{CKD}$ than hypertension. Our findings suggested reduced urate excretion as the more likely cause of hyperuricemia than excessive intake. Studies aimed at finding causal relationships are needed to ascertain definitive interactions.

\section{Acknowledgements}

We acknowledge the contribution and support of all the nurses and supporting staffs of the nephrology and hypertension clinic of Babcock University Teaching Hospital.

\section{Conflicts of Interest}

The authors declare no conflicts of interest regarding the publication of this paper.

\section{References}

[1] Mallat, S.G., Kattar, S.A., Tanios, B.Y. and Jurius, A. (2016) Hyperuricemia, Hypertension, and Chronic Kidney Disease: An Emerging Association. Current Hypertension Reports, 18, 74. https://doi.org/10.1007/s11906-016-0684-Z

[2] Stanifer, J.W., Jing, B., Tolan, S., Helmke, N., Mukerjee, R., Naicker, S., et al. (2014) The Epidemiology of Chronic Kidney Disease in Sub-Saharan Africa: A Systematic Review and Meta-Analysis. The Lancet Global Health, 2, e174-e181. https://doi.org/10.1016/S2214-109X(14)70002-6

[3] Mahamat, A.G., Hamat, I., Tondi, Z., Lemrabott, A., Faye, M., Moustapha, C., Sabi, K., Ka, K., Abdou, N. and Boucar, D. (2017) Hyperuricemia in Patients with Chronic Renal Failure in the General Hospital of National Reference of N'Djamena (Chad). Open Journal of Nephrology, 7, 9-18. https://doi.org/10.4236/ojneph.2017.71002

[4] Adejumo, O., Okaka, E., Okwuonu, C. and Ojogwu, L. (2016) Hyperuricemia in Predialysis Chronic Kidney Disease Patients in Southern Nigeria. Sahel Medical Journal, 19, 21-26. https://doi.org/10.4103/1118-8561.181890

[5] Doualla, M., Halle, M.P., Moutchia, J., Tegang, S. and Ashuntantang, G. (2018) Determinants of Hyperuricemia in Non-Dialysed Chronic Kidney Disease Patients in Three Hospitals in Cameroon. BMC Nephrology, 19, 169. https://doi.org/10.1186/s12882-018-0959-5

[6] Mutluay, R., Deger, S.M., Bahadir, E., et al. (2012) Uric Acid Is an Important Predictor for Hypertensive Early Atherosclerosis. Advances in Therapy, 29, 276-286. https://doi.org/10.1007/s12325-012-0006-Z

[7] Lottmann, K., Chen, X. and Schädlich, P.K. (2012) Association between Gout and All-Cause as Well as Cardiovascular Mortality: A Systematic Review. Current Rheumatology Reports, 14, 195-203. https://doi.org/10.1007/s11926-011-0234-2

[8] Kang, D.H. and Ha, S.K. (2014) Uric Acid Puzzle: Dual Role as Anti-Oxidant and 
Pro-Oxidant. Electrolytes \& Blood Pressure, 12, 1-6.

https://doi.org/10.5049/EBP.2014.12.1.1

[9] Spitsin, S. and Koprowski, H. (2010) Role of Uric Acid in Alzheimer's Disease. Journal of Alzheimer's Disease, 19, 1337-1338.

https://doi.org/10.3233/JAD-2010-1336

[10] Weisskopf, M.G., O’Reilly, E., Chen, H., Schwarzschild, M.A. and Ascherio, A. (2007) Plasma Urate and Risk of Parkinson's Disease. American Journal of Epidemiology, 166, 561-567. https://doi.org/10.1093/aje/kwm127

[11] Rentzos, M., Nikolaou, C., Anagnostouli, M., et al. (2006) Serum Uric Acid and Multiple Sclerosis. Clinical Neurology and Neurosurgery, 108, 527-531. https://doi.org/10.1016/j.clineuro.2005.08.004

[12] Nieto, F.J., Iribarren, C., Gross, M.D., Comstock, G.W. and Cutler, R.G. (2000) Uric Acid and Serum Antioxidant Capacity: A Reaction to Atherosclerosis? Atherosclerosis, 148, 131-139. https://doi.org/10.1016/S0021-9150(99)00214-2

[13] Chalmers, J., MacMahon, S., Mancia, G., Whitworth, J., Beilin, L., Hansson, L., et al. (1999) 1999 World Health Organization-International Society of Hypertension Guidelines for the Management of Hypertension. Guidelines Sub-Committee of the World Health Organization. Clinical and Experimental Hypertension, 21, 1009-1060. https://doi.org/10.3109/10641969909061028

[14] Benn, C.L., Dua, P., Gurrell, R., Loudon, P., Pike, A., Storer, R.I., et al. (2018) Physiology of Hyperuricemia and Urate-Lowering Treatments. Frontiers in Medicine, 5 , 160. https://doi.org/10.3389/fmed.2018.00160

[15] Liyanage, T., Ninomiya, T., Jha, V., Neal, B., Patrice, H.M. and Okpechi, I. (2015) Worldwide Access to Treatment for End-Stage Kidney Disease: A Systematic Review. The Lancet, 385, 1975-1982. https://doi.org/10.1016/S0140-6736(14)61601-9

[16] Feig, D.I., Soletsky, B. and Johnson, R.J. (2008) Effect of Allopurinol on Blood Pressure of Adoles-Cents with Newly Diagnosed Essential Hypertension: A Randomized Trial. JAMA, 300, 924-932. https://doi.org/10.1001/jama.300.8.924

[17] Lee, J.W. and Lee, K.H. (2019) Comparison of Renoprotective Effects of Febuxostat and Allopurinol in Hyperuricemic Patients with Chronic Kidney Disease. International Urology and Nephrology, 51, 467-473. https://doi.org/10.1007/s11255-018-2051-2

[18] National Kidney Foundation (2012) KDOQI Clinical Practice Guideline for Diabetes and CKD: 2012 Update. American Journal of Kidney Diseases, 60, 850-886. https://doi.org/10.1053/j.ajkd.2012.07.005

[19] Emamian, S.A., Nielsen, M.B., Pedersen, J.F., et al. (1993) Kidney Dimensions at Sonography: Correlation with Age, Sex, and Habitus in 665 Adult Volunteers. AJR. American Journal of Roentgenology, 160, 83-86. https://doi.org/10.2214/ajr.160.1.8416654

[20] Tsai, C.-W., Lin, S.-Y., Kuo, C.-C. and Huang, C.-C. (2017) Serum Uric Acid and Progression of Kidney Disease: A Longitudinal Analysis and Mini-Review. PLoS ONE, 12, e0170393. https://doi.org/10.1371/journal.pone.0170393

[21] Meng, L., Yu, W., Wang, T., Zhang, L., Heerdt, P. and Gelb, A.W. (2018) Blood Pressure Targets in Perioperative Care. Provisional Considerations Based on a Comprehensive Literature Review. BMJ Hypertension, 72, 806-817. https://doi.org/10.1161/HYPERTENSIONAHA.118.11688

[22] National Cholesterol Education Program (NCEP) Expert Panel on Detection, Evaluation, and Treatment of High Blood Cholesterol in Adults (Adult Treatment Panel III) (2002) Third Report of the National Cholesterol Education Program (NCEP) 
Expert Panel on Detection, Evaluation, and Treatment of High Blood Cholesterol in Adults (Adult Treatment Panel III) Final Report. Circulation, 106, 3143-3421. https://doi.org/10.1161/circ.106.25.3143

[23] Cappellini, M.D. and Mota, I. (2015) Anemia in Clinical Practice-Definition and Classification: Does Hemoglobin Change with Aging? Seminars in Hematology, 52, 261-269. https://doi.org/10.1053/j.seminhematol.2015.07.006

[24] Boag, A.M., Breheny, C., Handel, I. and Gow, A.G. (2019) Evaluation of the Effect of Urine Dip vs Urine Drip on Multi-Test Strip Results. Veterinary Clinical Pathology, 48, 276-281. https://doi.org/10.1111/vcp.12730

[25] Kim, S., McClave, S.A., Martindale, R.G., Miller, K.R. and Hurt, R.T. (2017) Hypoalbuminemia and Clinical Outcomes: What Is the Mechanism behind the Relationship? The American Surgeon, 83, 1220-1227. https://doi.org/10.1177/000313481708301123

[26] Levey, A.S., Stevens, L.A., Schmid, C.H., Zhang, Y.L., Feldman, H.I., Eggers, P., Van Lente, F., Greene, T., et al. (2009) A New Equation to Estimate Glomerular Filtration Rate. Annals of Internal Medicine, 150, 604-612. https://doi.org/10.7326/0003-4819-150-9-200905050-00006

[27] Cannon, P.J., Stason, W.B., Demartini, F.E., Sommers, S.C. and Laragh, J.H. (1966) Hyperuricemia in Primary and Renal Hypertension. NEJM, 275, 457-464. https://doi.org/10.1056/NEJM196609012750902

[28] Lin, C.S., Lee, W.L., Hung, Y.J., Lee, D.Y., Chen, K.F., Chi, W.C., et al. (2012) Prevalence of Hyperuricemia and Its Association with Antihypertensive Treatment in Hypertensive Patients in Taiwan. International Journal of Cardiology, 56, 41-46. https://doi.org/10.1016/j.ijcard.2010.10.033

[29] Liu, J., Chen, L., Yuan, H., Huang, K., Li, G., Sun, N., et al. (2021) Survey on Uric Acid in Chinese Subjects with Essential Hypertension (SUCCESS): A Nationwide Cross-Sectional Study. Annals of Translational Medicine, 9, 27. https://doi.org/10.21037/atm-20-3458

[30] Fan, X.H., Sun, K., Wang, Y.B., Dang, A.M., Zhou, X.L., Zhang, H.M., et al. (2009) Prevalence and Associated Risk Factors of Hyperuricemia in Rural Hypertensive Patients. Chinese Medical Journal, 89, 2667-2670.

[31] Emokpae, A. and Abdul, A. (2013) Serum Uric Acid Levels among Nigerians with Essential Hypertension. Nigerian Journal of Physiological Sciences, 28, 41-44.

[32] Alikor, C.A. and Emem-Chioma, P.C. (2013) Prevalence of Hyperuricaemia in a Rural Population in Nigeria Niger Delta Region. Nigerian Journal of Medicine, 22, 187-192.

[33] Makusidi, M.A., Chijioke, A., Kolo, P.M., Liman, H.M., AbdulRahman, M.B., Sani, B., et al. (2016) Prevalence and Pattern of Hyperuricemia in a Survey among Inhabitants of Sokoto Metropolis, North Western Nigeria. Research Journal of Health Sciences, 4, 1 .

[34] Wang, S.F., Shu, L., Wang, S., Wang, X.Q., Mu, M. and Hu, C.Q. (2014) Gender Difference in the Association of Hyperuricemia with Hypertension in a Middle-Aged Chinese Population. Blood Pressure, 23, 339-344.

https://doi.org/10.3109/08037051.2014.906131

[35] Grayson, P.C., Kim, S.Y., LaValley, M. and Choi, H.K. (2011) Hyperuricemia and Incident Hypertension: A Systematic Review and Meta-Analysis. Arthritis Care \& Research, 63, 102-110. https://doi.org/10.1002/acr.20344

[36] Ewenighi, C.O., Dimkpa, U., Ezeugwu, U., Onyeanusi, J., Onoh, L. and Adejumo, B. (2015) Prevalence of Hyperuricemia and Its Risk Factors in Healthy Male Adults 
from Abakaliki Metropolis, Nigeria. Journal of Molecular Pathophysiology, 4, 94-98. https://doi.org/10.5455/jmp.20150915011842

[37] Ulasi, I. (2008) Gender Bias in Access to Healthcare in Nigeria: A Study of End Stage Renal Disease. Tropical Doctor, 38, 50-52.

https://doi.org/10.1258/td.2007.060160

[38] Verzola, D., Gandolfo, M.T., Salvatore, F., Villaggio, B., Gianiorio, F., Traverso, P., et al. (2004) Testosterone Promotes Apoptotic Damage in Human Renal Tubular Cells. Kidney International, 65, 1252-1261. https://doi.org/10.1111/j.1523-1755.2004.00497.x

[39] Avram, Z. and Krishnan, E. (2008) Hyperuricaemia-Where Nephrology Meets Rheumatology. Rheumatology (Oxford), 47, 960-964.

https://doi.org/10.1093/rheumatology/ken070

[40] Stewart, D.J., Langlois, V. and Noone, D. (2019) Hyperuricemia and Hypertension: Links and Risks. Integrated Blood Pressure Control, 12, 43-62. https://doi.org/10.2147/IBPC.S184685

[41] Goicoechea, M., Garcia de Vinuesa, S., Verdalles, U., Verde, E., Macias, N., Santos, A., et al. (2015) Allopurinol and Progression of CKD and Cardiovascular Events: Long-Term Follow-up of a Randomized Clinical Trial. American Journal of Kidney Diseases: The Official Journal of the National Kidney Foundation, 65, 543-549. https://doi.org/10.1053/j.ajkd.2014.11.016

[42] Mehta, T., Nuccio, E., McFann, K., Madero, M., Sarnak, M.J. and Jalal, D. (2015) Association of Uric Acid with Vascular Stiffness in the Framingham Heart Study. American Journal of Hypertension, 28, 877-883. https://doi.org/10.1093/ajh/hpu253

[43] Kuwabara, M., Hisatome, I., Niwa, K., Hara, S., Roncal-Jimenez, C.A., Bjomstad, P. et al. (2018) Uric Acid Is a Strong Risk Marker for Developing Hypertension from Prehypertension: A 5-Year Japanese Cohort Study. Hypertension, 71, 78-86. https://doi.org/10.1161/HYPERTENSIONAHA.117.10370

[44] Mazza, A., Lenti, S., Schiavon, L., Monte, A.D., Townsend, D.M., Ramazzina, E., et al. (2017) Asymptomatic Hyperuricemia Is a Strong Risk Factor for Resistant Hypertension in Elderly Subjects from General Population. Biomedicine \& Pharmacotherapy, 86, 590-594. https://doi.org/10.1016/j.biopha.2016.11.104

[45] Imazu, M., Yamamoto, H., Toyofuku, M. and Sumii, K. (2002) Hyperinsulinemia for the Development of Hypertension: Data from the Hawaii-Los Angeles-Hiroshima Study. Hypertension Research, 24, 531-536.

https://doi.org/10.1291/hypres.24.531

[46] Biyik, Z. and Guney, I. (2018) Relationship between Uric Acid, Proteinuria, and Atherogenic Index of Plasma in Renal Transplant Patients. Transplantation Proceedings, 50, 3376-3380. https://doi.org/10.1016/j.transproceed.2018.05.021

[47] Jayasinghe, K., White, S.M., Kerr, P.G., MacGregor, D., Stark, Z., Wilkins, E., et al. (2019) Isolated Proteinuria Due to CUBN Homozygous Mutation-Challenging the Investigative Paradigm. BMC Nephrology, 20, 330.

https://doi.org/10.1186/s12882-019-1474-z

[48] McAdams-DeMarco, M.A., Maynard, J.W., Coresh, J. and Baer, A.N. (2012) Anemia and the Onset of Gout in a Population-Based Cohort of Adults: Atherosclerosis Risk in Communities Study. Arthritis Research \& Therapy, 14, R193. https://doi.org/10.1186/ar4026

[49] Kuwabara, M., Niwa, K., Nishi, Y., Mizuno, A., Asano, T., Masuda, K., et al. (2014) Relationship between Serum Uric Acid Levels and Hypertension among Japanese Individuals Not Treated for Hyperuricemia and Hypertension. Hypertension Re- 
search, 37, 785-789. https://doi.org/10.1038/hr.2014.75

[50] Kuwabara, M., Niwa, K., Nishi, Y., Hisatome, I., et al. (2011) The Positive Relationship between Uric Acid and Hypertension in Japanese People Not Taking Antihypertensive Drugs. Journal of Hypertension, 29, e32-e33.

https://doi.org/10.1097/01.hjh.0000408079.36559.0f

[51] Bulbul, M.C., Dagel, T., Afsar, B., Ulusu, N.N., Kuwabara, M., Covid, A., et al. (2018) Disorders of Lipid Metabolism in Chronic Kidney Disease. In-Depth Review. Blood Purification, 46, 144-152. https://doi.org/10.1159/000488816 